\title{
Régulations métaboliques de Lactococcus lactis en culture pure ou mixte dans le lait
}

\author{
Sophie Drouault ${ }^{\mathrm{a}}$, Gérard Corthier ${ }^{\mathrm{b}}$, Christine Delorme ${ }^{\mathrm{a}}$, \\ S. Dusko Ehrlich ${ }^{\text {a }}$, Pierre Renault ${ }^{\mathrm{a} *}$
}

${ }^{a}$ Laboratoire de génétique microbienne, Inra, 78352 Jouy-en-Josas cedex, France

${ }^{b}$ Unité d'écologie et de physiologie du système digestif, Inra, 78352 Jouy-en-Josas cedex, France

\begin{abstract}
Metabolic regulation of Lactococcus lactis grown in pure or co-culture in milk. The cell responds to environmental changes by triggering or repressing the expression of its genes in order to adapt its metabolism to the new conditions. The measure of promoter activities could thus allow an indirect assessment of the external signals that the cell has integrated and the modification of its metabolic potential. We have developed a method using this idea to evaluate the bacterial metabolism independently of its externalized products. Promoter activity is measured by following the expression of luciferases as reporter genes. Two different luciferase genes were used, luxAB from the bacteria Vibrio harveyi and luc from the eucaryote Photinus pyralis. The activity of the procaryotic and the eucaryotic enzymes is detectable by on-line measurement with whole cells when the cells provide the cofactors FMNH and ATP, respectively. This method is very sensitive, allowing the detection of weak promoter activity, or moderate transcription at low cell density. To demonstrate that this method is efficient, we studied promoter activities modulated by the presence of available amino acids with bacterial culture in milk. This allows us to see when the cells are starving, either in pure cultures or in mixed cultures with competing bacteria. As the two luciferases can be detected independently in the same culture, this method should allow the study of the interaction between strains in coculture at the molecular level. (C) Inra/Elsevier, Paris.
\end{abstract}

metabolism / luciferase / metabolic probe / mixte culture / Lactococcus lactis/ milk

Résumé - La cellule répond aux changements de condition de son milieu en activant ou réprimant de façon coordonnée l'expression de ses gènes pour adapter son métabolisme. La mesure de l'activité des promoteurs pourrait donc permettre de connaître indirectement la perception que la cellule a du milieu et la manière dont elle va répondre. Nous avons donc conçu une méthode fondée sur ce principe pour évaluer le métabolisme cellulaire, indépendamment de la mesure de ses produits. La mesure des activités promotrices se fait à l'aide de gènes rapporteurs émetteurs de lumière, luxAB de Vibrio harveyi, ou luc de Photinus pyralis. La détection de l'activité des luciférases est simple à mettre en œuvre et très sensible. De plus, elle peut être réalisée dans des milieux complexes comme le lait. La variation de l'activité de promoteurs régulés par

\footnotetext{
* Correspondance et tirés à part
} 
la présence ou l'absence d'acides aminés permet ainsi de révéler le moment où le milieu est carencé au cours d'une cinétique de croissance. Cette méthode peut s'appliquer à l'étude de cellules en culture pure, mais aussi en coculture. Il est alors possible d'étudier le métabolisme d'une souche au sein d'un écosystème complexe pour mieux comprendre les phénomènes de compétition ou de symbiose dans les levains. (C) Inra/Elsevier, Paris.

métabolisme / luciférase / sonde / culture mixte / Lactococcus lactis / lait

\section{INTRODUCTION}

Les lactocoques sont largement utilisés dans les fermentations lactiques. L'amélioration des souches nécessite une meilleure compréhension de leur métabolisme et des facteurs qui contrôlent l'expression des gènes impliqués lors de ces fermentations. Les régulations génétiques permettent de coordonner les fonctions bactériennes. Jusqu'à présent seules quelques unes de ces régulations ont été étudiées et ce, en milieu de laboratoire. Cependant les résultats ainsi obtenus ne sont pas forcément extrapolables à d'autres milieux tels que le lait. Pour développer l'étude des bactéries dans leur écosystème, il faut disposer de méthodes efficaces pour mesurer l'expression des gènes dans le lait si l'on étudie des levains lactiques.

La luciférase bactérienne de Vibrio harveyi, enzyme productrice de lumière, peut être utilisée comme gène rapporteur chez les bactéries lactiques $[6,22]$. Le dosage de l'activité de cette enzyme est très sensible et permet de mesurer l'activité de promoteurs peu actifs, ainsi que des activités à des densités cellulaires faibles [13, 24]. Dans cet article, nous avons montré que les gènes codant pour les luciférases bactérienne et eucaryote, couplés à différents promoteurs répondant à des carences en acides aminés permettaient de détecter leur carence dans le milieu au cours de la croissance. Ces mesures peuvent être réalisées en culture pure mais aussi spécifiquement sur une souche poussant en association avec d'autres bactéries, ce qui permet de sonder l'état physiologique des cellules marquées. Cette méthode est plus sensible, plus spécifique et plus facile à mettre en œuvre que les méthodes basées sur l'utilisation de sondes fluorescentes en microscopie ou en cytométrie de flux $[1,3]$. Enfin, un système à deux gènes rapporteurs indépendants a été mis au point et permet de suivre plusieurs paramètres dans des cultures mixtes.

\section{LES LUCIFÉRASES, DES GẼNES RAPPORTEURS ADAPTÉS À L'ÉTUDE DES ÉCOSYSTĖMES COMPLEXES}

Il existe chez certains organismes des phénomènes d'émission de lumière [12]. Ces processus ont été dénommés bioluminescences et les enzymes responsables de la production de lumière, luciférases. Ces réactions peuvent être catalysées par des enzymes bâties selon différentes architectures selon leur organisme d'origine. Les luciférases oxydent en général un substrat, ce qui s'accompagne d'émission de lumière. Dans ce travail, nous utiliserons deux types d'enzymes ayant des substrats différents. Le premier système est bactérien, issu de la bactérie luminescente Vibrio harveyi, et requière un aldéhyde à longue chaîne carbonée, et la flavine mononucléotide réduite $\left(\mathrm{FMNH}_{2}\right)$ dont l'oxydation provoque une réaction de luminescence [19]. La réaction peut être schématisée par : 


$$
\begin{aligned}
& \mathrm{FMNH}_{2}+\mathrm{R}-\mathrm{CO}-\mathrm{H}+\mathrm{O}_{2} \\
& \stackrel{\mathrm{LuxA}-\mathrm{LuxB}}{\longrightarrow} \mathrm{FMN}+\mathrm{R}-\mathrm{COOH}+\mathrm{H}_{2} \mathrm{O} \\
& \text { + lumière }(490 \mathrm{~nm})
\end{aligned}
$$

Le second système est issu du vers luisant (Photinus pyralis). Dans cette réaction le substrat luminescent, la luciférine, est activé par l'ATP en présence de $\mathrm{Mg}^{2+}$ pour former un complexe luciférine-luciférase. La luciférine du complexe est alors oxydée et revient à un état stable en émettant un photon [12]. L'ATP est donc nécessaire pour fournir l'énergie à la première phase de la réaction qui peut se résumer par :

$$
\begin{gathered}
\mathrm{ATP}+\mathrm{O}_{2}+\mathrm{D} \text {-luciférine } \\
\stackrel{\mathrm{Luc}}{\longrightarrow} \mathrm{AMP}+\mathrm{PPi}+\mathrm{CO}_{2}+\text { oxyluciférine } \\
+ \text { lumière }(560 \mathrm{~nm})
\end{gathered}
$$

Avec ces deux systèmes, l'émission de lumière est mesurée avec un luminomètre directement à partir des cellules en suspension sans extraction préalable. En effet, les substrats diffusent à travers les membranes, et les cellules fournissent le $\mathrm{FMNH}_{2}$ ou l'ATP requis pour les réactions. Il en résulte que la mesure de l'expression de ces gènes rapporteurs ne peut se faire que dans des cellules énergétiquement actives, et donc capables de répondre aux changements de l'environnement. Étant donné la différence des réactions chimiques des deux luciférases, nous verrons qu'il est possible de discriminer leurs activités par l'addition de leur substrat respectif, et donc de les mesurer dans les mêmes cultures indépendamment l'une de l'autre, étendant ainsi les possibilités d'investigations à deux promoteurs dans une même souche ou à deux souches en culture mixte.

\section{DES PROMOTEURS POUR ÉTUDIER LES BESOINS EN ACIDES AMINÉS DES BACTÉRIES}

L'un des paramètres limitant la croissance des bactéries lactiques dans le lait est la disponibilité d'une source d'acides aminés assimilables [15]. Ainsi les souches de L. lactis ne possédant pas de protéase de paroi ne poussent qu'à un niveau d'environ $10 \%$ par rapport à celles qui assimilent les caséines. Parmi les acides aminés souvent cités comme limitant, se trouvent les acides aminés branchés (isoleucine, leucine et valine, ILV) et l'histidine [5, 9]. Les gènes codant pour la biosynthèse de ces acides aminés ont été caractérisés et leur régulation étudiée en détail $[4,8,10$, 23] (voir aussi Delorme et al., 1997, soumis pour publication ; ces résultats sont résumés dans le tableau I). En principe, l'expression de ces gènes devrait être activée dans le lait lorsque les acides aminés assimilables sont épuisés. La mesure de l'activité des promoteurs contrôlant ces gènes devrait donc nous renseigner directement sur le niveau de carence de ces acides aminés lors de la croissance bactérienne.

Les promoteurs d'opérons de biosynthèse des acides aminés utilisés dans ce travail ont été isolés de la souche de L. lactis subsp lactis NCDO 2118 (tableau I). Le promoteur histidine $\left(\mathrm{P}_{\text {his }}\right)$ qui contrôle 1'opéron de biosynthèse de l'histidine est réprimé en milieu riche et ouvert en carence d'histidine. Nous avons construit une forme dérivée de ce promoteur $\left(\mathrm{P}_{\text {his }}\right)$ qui n'est plus régulée par l'histidine. Son activité est élevée et semblable en présence et en absence d'histidine (Delorme et al., 1997, soumis pour publication). $\mathrm{P}_{\text {hiss }}$ a été choisi initialement dans cette étude comme promoteur constitutif. La biosynthèse des acides aminés branchés est contrôlée par les promoteurs $\mathrm{P}_{\text {leu }}$ et $\mathrm{P}_{i l v}$ [11]. Ils sont réprimés en milieu riche et ouverts en carence d'isoleucine. Le dernier promoteur, celui contrôlant l'expression de l'acétolactate décarboxylase $\left(\mathrm{P}_{\text {aldB }}\right)$ n'est pas impliqué dans l'expression de gène de biosynthèse d'acides aminés. Cependant il permet l'expression de deux 
Tableau I. Activité des promoteurs de L. lactis NCDO2118 (Lac-, Prt). Les mesures ont été réalisées en milieu chimiquement défini (MCD) [20] et dans le lait. Les gènes codant pour la luciférase de Vibrio harveyi (luxAB) sont placés sous contrôle des différents promoteurs et intégrés dans le chromosome. L'activité luciférase est mesurée à l'aide d'un luminomètre après addition du substrat (nonaldéhyde) à la culture bactérienne. Le pic d'émission est pondéré par la population bactérienne. L'activité luciférase est exprimée en lux pour $10^{8} \mathrm{CFU}$.

Table I. Promoter activity in L. lactis NCDO2118 (Lac-, Prt). Measure of luciferase activity was performed on cells grown in chemical defined medium (MCD) [20] and in milk luxAB genes from Vibrio harveyi are transcribed under the control of several promoters and the construction was inserted in the chromosome. Luciferase activity is determined with a luminometer after addition to a culture sample of nonaldehyde as substrate. The value corresponding to the pick of emitted light is reported to the number of cells and expressed as lux per $10^{8} \mathrm{CFU}$.

\begin{tabular}{llrrrr}
\hline & & \multicolumn{3}{c}{ Luciférase / L. lactis $\times 10^{8}$} \\
\cline { 3 - 6 } & & $\mathrm{P}_{\text {his }}$ & $\mathrm{P}_{\text {hiss }}$ & $\mathrm{P}_{\text {leu-ilv }}$ & $\mathrm{P}_{\text {aldB }}$ \\
\cline { 3 - 6 } MCD & Carence en acides aminés & & \\
& & $\mathrm{ND}^{\mathrm{b}}$ & $\mathrm{ND}$ & $\mathrm{ND}$ & 24000 \\
& Carence en ILV & $\mathrm{ND}$ & $\mathrm{ND}$ & 6200 & 22000 \\
& Carence en histidine & 3300 & 23000 & $\mathrm{ND}$ & 30000 \\
& Pas de carence & 280 & 26000 & 1600 & 55000 \\
Lait & Phase exponentielle $(5 \mathrm{~h})$ & 500 & 25000 & 1000 & 50000 \\
& Phase stationnaire $(20 \mathrm{~h})$ & 700 & 140000 & 85000 & 30000 \\
& & & & &
\end{tabular}

${ }^{a}$ Acides aminés testés : sérine, méthionine, isoleucine, leucine et valine. ${ }^{b}$ Non déterminés.

${ }^{a}$ Aminoacid tested, serine, methionine, isoleucine, leucine and valine. ${ }^{b}$ Not determined.

gènes ayant des rôles de régulateurs dans le métabolisme des acides aminés et du carbone [11]. Il est ouvert en milieu riche et partiellement fermé dans des conditions de réponse stringente dues par exemple à la carence en certains acides aminés [10]. Enfin, un dernier type de promoteur a été utilisé, $\mathrm{P}_{\text {orfD-repE }}-\mathrm{P}_{\text {res }}$. Il contrôle la réplication de $\mathrm{pAM} \beta 1$ et de ses dérivés $[17,18]$. Son activité permet de maintenir un nombre de plasmides constant par cellule.

\section{4. ÉTUDE D'UNE SOUCHE LAC-, PRT-EN MILIEU LAIT}

Les souches de $L$. lactis qui ne possèdent pas le plasmide avec les gènes $l a c_{P T S}$ (nécessaires à l'hydrolyse rapide du lactose) et prtPM (protéases de paroi) se développent mal dans le lait. Cependant certaines souches telles que la NCDO2118, possèdent une lactase chromosomique qui leur permet de se multiplier dans le lait jusqu'à un niveau de $10^{8} \mathrm{UFC} / \mathrm{mL}$. Leur croissance est lente et accompagnée d'une faible acidification du lait.

Dans le lait, toutes les souches dérivées de L. lactis NCDO2118 qui contiennent les différentes constructions génétiques, promoteur - $\operatorname{lu} x A B$, ont une croissance semblable. Elles atteignent un plateau au bout de 7 heures pour effectuer un ultime doublement entre 7 et 14 heures (figure I). Le tableau I et la figure 1 montrent l'activité des promoteurs décrits précédemment lors de cultures dans le lait. Les promoteurs $\mathrm{P}_{\text {leu-ilv }}$ sont fermés dans une première phase en milieu lait. Cependant après 12 heures de croissance, ils sont activés pour atteindre après 20 heures de culture un niveau proche de celui observé en milieu chimiquement défini (MCD) 
Figure 1. Croissance et activités luciférases de $L$. lactis dans le lait. Les différentes souches portent sur le chromosome les gènes codant pour la luciférase de Vibrio harveyi couplés aux promoteurs présentés dans le tableau I.

Figure 1. Growth and luciferase activity of L. lactis in milk. The different strains harbor in their chromosome the lux genes of $V$ harveyi under the control of the promoters presented in table $I$.

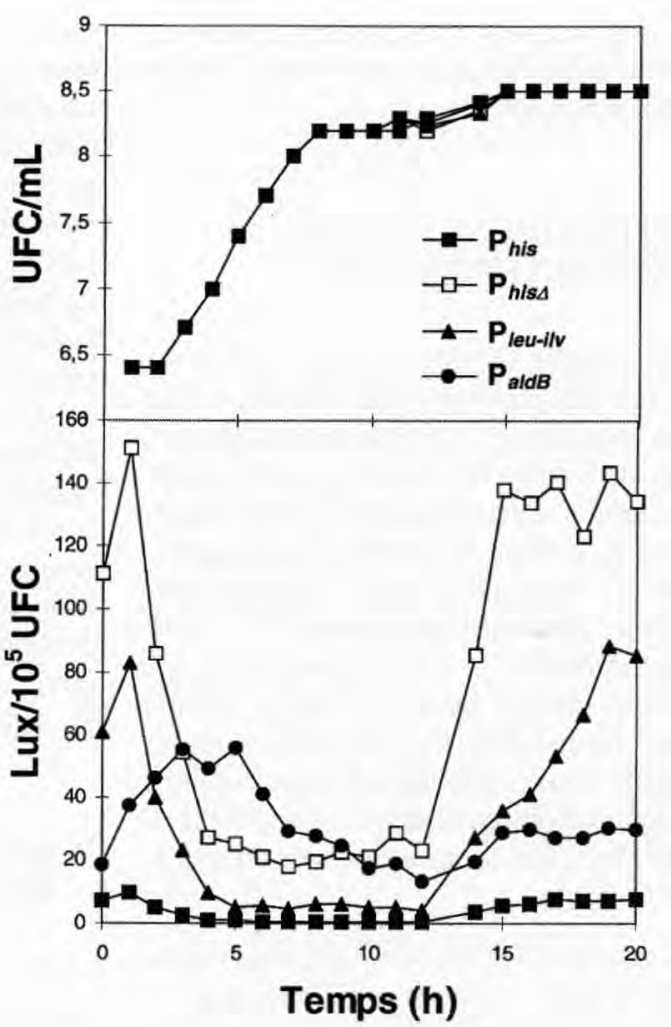

vité après 5-6 heures de croissance suggère donc que le milieu commence à être carencé en au moins un acide aminé. Une telle carence pourrait déclencher un mécanisme de régulation général, la réponse stringente. Chez les bactéries où cette régulation a été bien étudiée, elle a pour effet de bloquer de nombreux promoteurs et de provoquer ainsi un arrêt de la croissance bactérienne [2, 21]. Cependant, pendant cette réponse, les promoteurs des gènes de biosynthèse des acides aminés sont en général activés non spécifiquement. L'activation de $\mathrm{P}_{\text {his }}$, délété des séquences de contrôle spécifique à la réponse à une carence en histidine semble refléter ce phénomène lors de l'arrêt définitif de croissance. Il est en effet très actif immédiatement après l'ensemencement, puis se ferme dès le démarrage de la croissance et redevient actif après 15 heures 
de culture. Son activité maximale en milieu lait est 6 fois supérieure à celle observée en MCD.

\section{5. ÉTUDE D'UNE SOUCHE EN CULTURE MIXTE}

Les gènes luciférases permettent de suivre l'état physiologique d'une bactérie dans un milieu où il est en général très difficile de récolter les bactéries pour réaliser des études métaboliques directes (composition, dosage de composés intracellulaires...). Nous allons voir ici que ces marqueurs cellulaires permettent de suivre spécifiquement le métabolisme dans des cultures mixtes. Dans un premier temps, nous avons testé l'effet d'une souche capable d'utiliser le lactose et les caséines sur le métabolisme de la souche NCDO2118 ( $\mathrm{Lac}^{-}, \mathrm{Prt}$ ) lors d'une croissance en coculture,

La souche $\mathrm{Prt}^{+}$hydrolyse la caséine en de nombreux peptides [14]. Elle pourrait éventuellement fournir ainsi des acides aminés à la souche $\operatorname{Prt}$ [16]. Le suivi de croissance montre qu'en culture mixte, la souche protéase négative accuse un léger retard de croissance par rapport aux expériences où elle pousse en culture pure. De plus, l'activité luciférase sous contrôle des promoteurs utilisés comme sondes métaboliques est différente. Les changements les plus significatifs se produisent au cours de la croissance entre 3 et 5 heures, et non pendant la phase de latence. Toujours par comparaison avec une situation de culture pure, en culture mixte les promoteurs $\mathrm{P}_{\text {his }}$ et $\mathrm{P}_{\text {ald } B}$ sont alors plus actifs (figure 2). Nous avons vu précédemment que (i) l'activation de $\mathrm{P}_{\text {his }}$ était probablement le résultat de la carence de la cellule en certains acides aminés ou de l'entrée des cellules en phase stationnaire et que (ii) 1'activité de $\mathrm{P}_{\text {aldB }}$ diminuait dans des conditions de carence en acides aminés. Paradoxalement, il semble qu'en culture mixte l'acti- vité de ce dernier se maintienne à un niveau supérieur à celui des cultures pures. La différence de réponse donnée par ces deux marqueurs pourrait s'expliquer s'ils ne répondaient pas aux mêmes carences. Une étude plus poussée des facteurs régulant $\mathrm{P}_{\text {his }}$ et $\mathrm{P}_{\text {aldB }}$ devrait nous permettre de mieux comprendre le métabolisme de L. lactis dans le lait. Il est cependant établi que la présence de la souche $\mathrm{Lac}^{+} \mathrm{Prt}^{+}$ modifie significativement la physiologie de la souche $\mathrm{Lac}^{-} \mathrm{Prt}$, probablement par le biais d'une modification du milieu en acides aminés assimilables. Cette étude éclaire sous un jour différent les interactions observées entre des souches possédant ou pas une protéase de paroi et devrait être étendue à d'autres types de souches $[7,16]$.

\section{6. ÉTUDE SIMULTANÉE DE DEUX SOUCHES EN CULTURE MIXTE}

Nous avons vu précédemment qu'il existait plusieurs luciférases dont les activités pouvaient être mesurées par l'addition du substrat approprié. Dans un premier temps nous avons placé le gène luc sous contrôle de signaux d'expression fonctionnels chez les lactocoques (site de fixation des ribosomes, promoteurs). La luminescence émise par ce système est, à promoteur égal, environ dix fois moindre que celle obtenue avec la luciférase bactérienne. Cependant, les activités mesurées restent à un niveau bien supérieur au bruit de fond. Enfin, les résultats (induction, niveau relatif d'expression...) obtenus avec différents promoteurs en fusion avec luc sont comparables à ceux réalisés avec lux $A B$.

La possibilité d'utiliser ces deux gènes rapporteurs ensemble dans la même expérience a été testée sur des cultures mixtes. La figure 3 montre le résultat obtenu lors d'une culture mixte dans le lait avec deux souches de lactocoque, l'une exprimant 


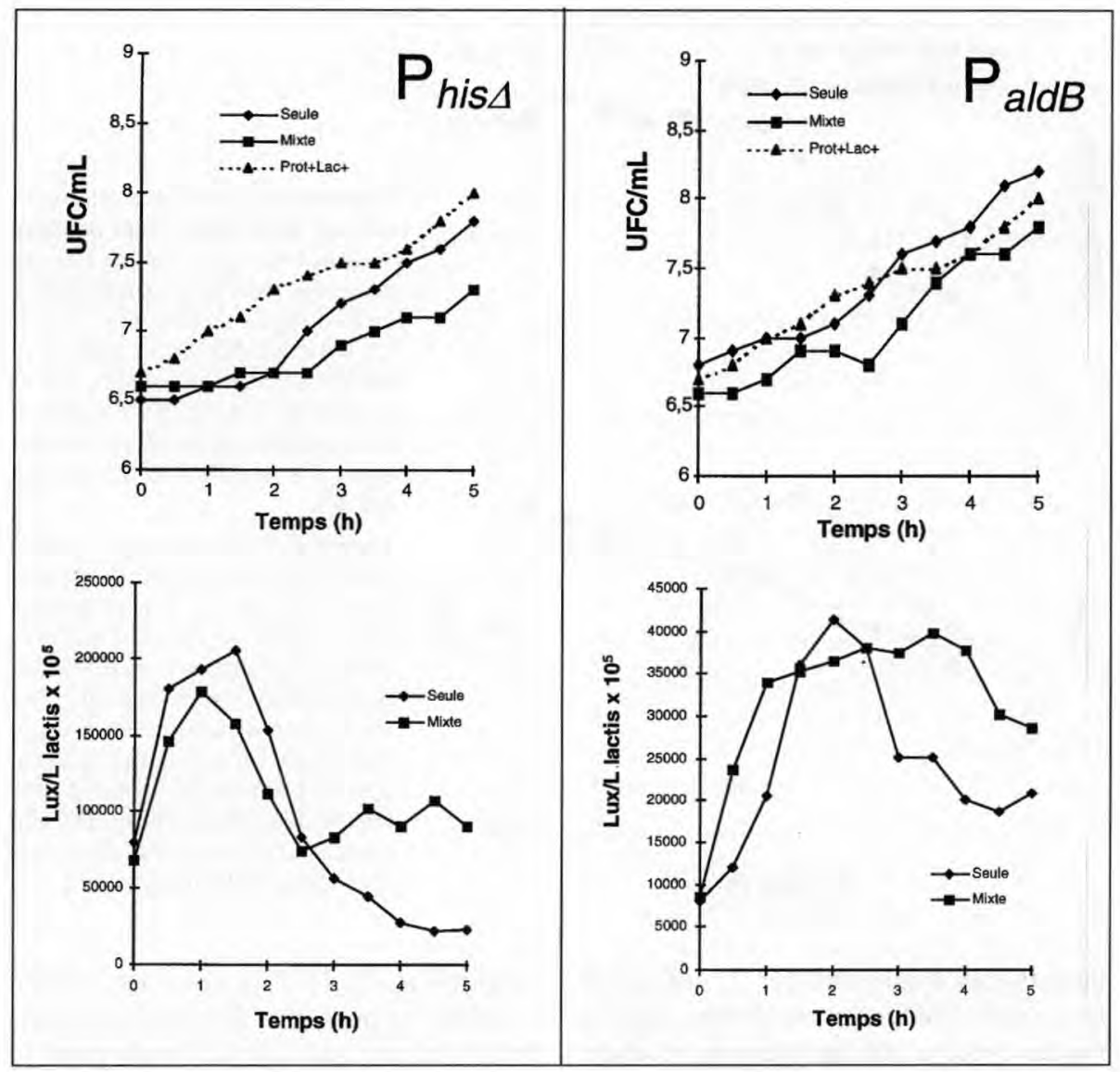

Figure 2. Croissance et activités luciférases de souches de L. lactis NCDO2118 (Lac-, Prt-) dans le lait seule ou en association avec la souche NCDO763 $\left(\mathrm{Lac}^{+}, \mathrm{Prt}^{+}\right)$. Les gènes codant pour la luciférase de Vibrio harveyi couplés aux promoteurs $\mathrm{P}_{\text {his }}$ et $\mathrm{P}_{\text {aldB }}$ sont insérés dans le chromosome. Les cultures mixtes sont réalisée en association avec la souche NCDO763 $\left(\mathrm{Lac}^{+}, \mathrm{Prt}^{+}\right)$.

Figure 2. Growth and luciferase activity of derivatives of L. lactis NCDO2118 ( $\left.\mathrm{Lac}^{-}, \mathrm{Prt}^{-}\right)$ grown in milk alone or in association with the strain NCDO763 $\left(\mathrm{Lac}^{+}, \mathrm{Prt}^{+}\right)$. L. lactis NCDO2118 strains harbor in the chromosome the lux genes under the control of promoters $\mathrm{P}_{\text {his }}$ et $\mathrm{P}_{\text {ald }}$.

la luciférase bactérienne et l'autre la luciférase de luciole. Nous poursuivons actuellement ce type d'expérience pour mesurer l'effet de la présence de différentes souches, capables d'utiliser différemment le lactose ou les protéines du lait, sur le métabolisme de souches de différents phénotypes. Nous espérons ainsi mettre en évidence les effets sur le métabolisme des symbioses ou des compétitions lors des cocultures.

\section{CONCLUSION}

Cette étude montre que l'on dispose actuellement d'outils génétiques qui permettent d'étudier la régulation de promoteurs de L. lactis dans un milieu aussi complexe que le lait. Ces promoteurs peuvent révéler l'état physiologique de la cellule lorsqu'ils répondent à la présence ou carence d'un métabolite, ou à des condi- 


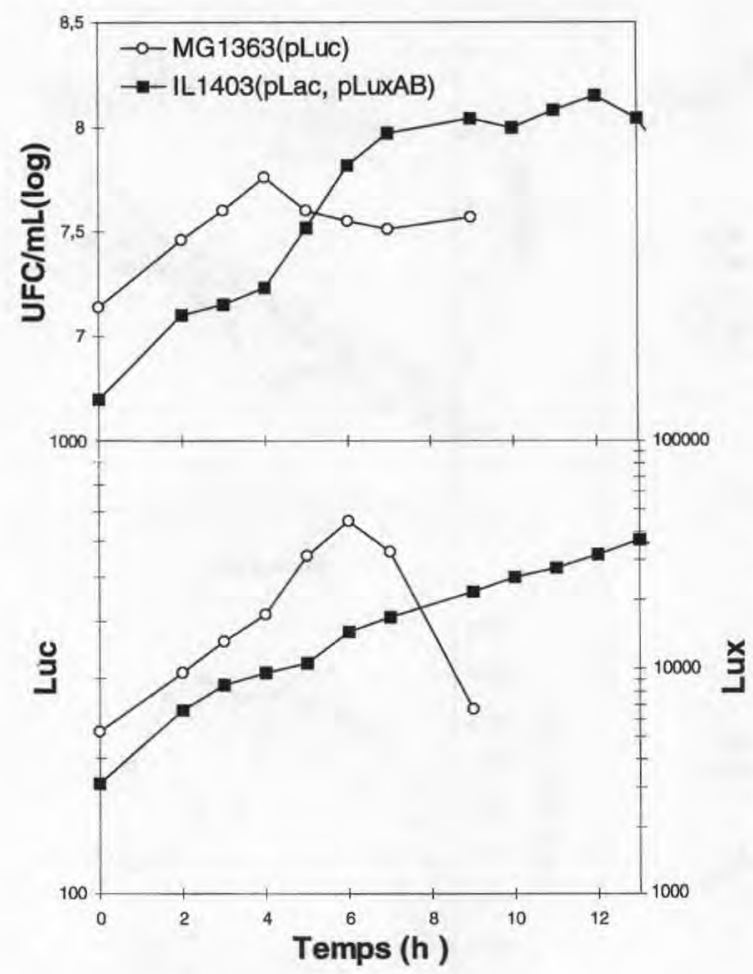

Figure 3. Croissance (haut) et activité lumineuse (bas) de deux souches de L. lactis cultivées ensemble dans le lait supplémenté en hydrolysat de caséines $(1 \mathrm{~g} / \mathrm{L})$. La souche MG1363 exprime la luciférase de la luciole (pLuc), et la souche IL1403, la luciférase bactérienne (pLuxAB) et le système d'assimilation du lactose (pLac).

Figure 3. Growth (upper panel) and luciferase activity (lower panel) of two L. lactis strains grown alone or in association in milk supplemented with casein hydrolysate ( $1 \mathrm{~g} / \mathrm{L})$. The MG1363 strain expresses the eucaryotic luciferase on a plasmid (pLuc). The IL1403 strain expresses the bacterial luciferase (pLuxAB) and contains a plasmid with the genes for lactose utilization ( $\mathrm{pLac}$ ). tions métaboliques définies. L'utilisation de « l'outil luciférase » dans des cultures mixtes montre que les interactions entre souches influent sur la régulation de certains promoteurs de la biosynthèse des acides aminés. Des études plus détaillées devraient permettre de savoir par des mesures directes, par exemple, ce qu'une souche contenant la protéase de paroi prélève et apporte comme source d'acides aminés assimilables à une souche qui en est dépourvue. Cet outil est actuellement le seul, à notre connaissance, qui permette une mesure du métabolisme d'une souche spécifique en coculture avec des souches génétiquement semblables (espèce, sous espèce, souches). Elle nécessite cependant d'introduire dans la souche des gènes rapporteurs. Ceux-ci ne semblent pas modifier significativement le métabolisme des souches, contrairement à la plupart des sondes utilisées en analyse d'image ou en cytométrie [1,3]. Elle présente comme avantage majeur, une plus grande sensibilité, et une spécificité totale pour la souche marquée. Enfin dans l'avenir certaines améliorations de souches se feront en contrôlant l'expression de certains gènes ou en exprimant de nouveaux gènes d'intérêt dans le lait. Le choix des promoteurs à employer pour cela sera crucial. La méthodologie que nous proposons permet de déterminer la force des promoteurs directement dans le lait et en fonction de la phase de croissance. Elle permettra donc d'orienter ce choix de manière fiable.

\section{RÉFÉRENCES}

[1] Amann R.I., Ludwig W., Schleifer K.H., Phylogenetic identification and in situ detection of individual microbial cells without cultivation, Microbiol. Rev. 59 (1995) 143-169. 
[2] Cashel M., Gentry D,R., Hernandez J., Vinella D., The stringent response. In: Escherichia coli and Salmonella typhimurium: $\mathrm{cel}$ lular and molecular biology, vol. 1 (F.C. Neidhardt, ed.), American Society for Microbiology, Washington D.C., (1996) 1458-1496.

[3] Davey H.M., Kell D.B., Flow cytometry and cell sorting of heterogeneous microbial populations: the importance of single-cell analyses, Microbiol. Rev. 60 (1996) 641-696.

[4] Delorme C., Ehrlich S.D., Renault P., Histidine biosynthesis genes in Lactococcus lactis subsp lactis, J. Bacteriol. 174 (1992) 6571-6579.

[5] Delorme C., Godon J.J., Ehrlich S.D., Renault P., Gene inactivation in Lactococcus lactis - Histidine biosynthesis, J. Bacteriol. 175 (1993) 4391-4399.

[6] Eaton T., Shearman C., Gasson M., The use of bacterial luciferase genes as reporter genes in Lactococcus: regulation of the Lactococcus lactis subsp. lactis lactose genes, J. Gen. Mierobiol. 139 (1993) 1495-1501.

[7] Flambard B., Richard J., Juillard V., Interaction between proteolytic strains of Lactococcus lactis influenced by different types of proteinase during growth, Appl. Environ. Microbiol. 63 (1997) 2131-2135.

[8] Godon J.J., Chopin M.C., Ehrlich S.D., Branched-chain amino acid biosynthesis genes in Lactococcus lactis subsp. lactis, J. Bacteriol. 174 (1992) 6580-6589.

[9] Godon J.J., Delorme C., Bardowski J., Chopin M.C., Ehrlich S.D., Renault P., Gene inactivation in Lactococcus lactis - Branchedchain amino acid biosynthesis, J. Bacteriol. 175 (1993) 4383-4390.

[10] Goupil N., Rôle de l'alpha-acétolactate décarboxylase chez Lactococcus lactis et régulation de sa synthèse $\mathrm{Ph} \mathrm{D}$ thesis University of Paris XI, Paris (1996)

[11] Goupil-Feuillerat N., Godon J.J., CocaignBousquet M., Ehrlich S.D., Renault P., Dual role of alpha-acetolactate decarboxylase in Lactococcus lactis subsp lactis, J. Bacteriol. 179 (1997) 6285-6293.

[12] Hastings J.W., Chemistries and colors of bioluminescent reactions: a review, Gene 173 (1996) 5-11.

[13] Hill P.J., Rees C.E.D., Winson M.K., Stewart G.S.A.B., The application of lux genes, Biotechnol. Appl. Biochem. 17 (1993) 3-14.
[14] Juillard V., Laan H., Kunji E.R.S., Jeronimusstratingh C.M., Bruins A.P., Konings W.N., The extracellular P-I-type proteinase of Lactococcus lactis hydrolyzes beta-casein into more than one hundred different oligopeptides. J. Bacteriol. 177 (1995a) 3472-3478.

[15] Juillard V., Le Bars D., Kunji E.R.S., Konings W.N., Gripon J.C., Richard J., Oligopeptides are the main source of nitrogen for Lactococcus lactis during growth in milk, Appl. Environ. Microbiol. 61 (1995b) 3024-3030.

[16] Juillard V., Furlan S., Foucaud C., Richard J., Mixed cultures in milk of a protease-positive and a protease-negative strains of Lactococcus lactis in milk, J. Dairy Sci. 79 (1996) 964-970.

[17] Lechatelier E., Ehrlich S.D., Janniere L., The pAMbetal CopF repressor regulates plasmid copy number by controlling transcription of the repE gene, Mol. Microbiol. 14 (1994) 463-471.

[18] Lechatelier E., Ehrlich S.D., Janniere L., Countertranscript-driven attenuation system of the pAMbeta1 repE gene. Mol. Microbiol. 20 (1996) 1099-1112.

[19] Meighen E.A., Molecular biology of bacterial luminescence. Microbiol. Rev. 55 (1991) 123-142.

[20] Poolman B., Konings W.N., Relation of growth of Streptococcus lactis and Streptococcus cremoris to amino acid transport, J. Bacteriol. 170 (1988) 700-707.

[21] Rallu F., Gruss A., Maguin E., Lactococcus lactis and stress. Antonie Van Leeuwenhoek 70 (1996) 243-251.

[22] Renault P., Corthier G., Goupil N., Delorme C., Ehrlich S.D., Plasmid vectors for Gram-positive bacteria switching from high to low copy number, Gene 183 (1996) 175-182.

[23] Renault P., Godon J.J., Goupil N., Delorme C. Corthier G., Ehrlich S.D., Metabolic operons in Lactococci. In: Genetics of Streptococci, Enterococci and Lactococci (Ferretti J.J., Gilmore M.S., Klaenhammer T.R.F., Brown, eds), Dev. Biol, Stand. Basel, Karger, SW 85 (1995) 431-441.

[24] Stewart G.S.A.B., Williams P., Lux genes and the applications of bacterial bioluminescence. J. Gen. Microbiol. 138 (1992) 1289-1300. 